\title{
Influência da idade na percepção de finitude e qualidade de vida
}

\author{
Influence of age on the perception of finitude and quality of life
}

Camila da Cruz Ramos de Araujo ${ }^{1}$

Adriana Coutinho do Azevedo Guimarães ${ }^{1}$

Carolina Meyer ${ }^{1}$

Leonessa Boing ${ }^{1}$

Manoella de Oliveira Ramos ${ }^{1}$

Melissa de Carvalho Souza ${ }^{1}$

Silvia Rosane Parcias ${ }^{1}$
${ }^{1}$ Centro de Ciências da Saúde e do Esporte,

Universidade do Estado de Santa Catarina. Av. Madre Benvenuta 2007, Itacorubi. 88.035-001 Florianópolis SC.cami.ramosdearaujo@ hotmail.com

\begin{abstract}
This cross-sectional study analyzed the influence of age on the perception of finitude and quality of life (QOL) of government employees, with a non-probabilistic accessibility sample composed of 471 employees with an average age of 40 . A self-administered questionnaire was used for the study. The results show that physical activity of group $C$ had the highest average in all components and group $B$ the lowest values, with the exception of walking. All the participants achieved good scores in the four domains of QOL (58\% $74 \%)$. In the perception of finitude, group Bobtained better scores (positive perception) in the four areas, whereas groups $A$ and $C$ had a more negative perception, there being no significant difference between the variables. This study reveals the positive relation of finitude in the age group from 35 to 50. Positive QOL and a high level of physical activity were identified.
\end{abstract}

Key words Finitude, Quality of life, Age
Resumo Estudo de corte transversal que analisou a influência da idade na percepção de finitude e qualidade de vida $(\mathrm{QV})$ de funcionários públicos, com uma amostra não probabilística por acessibilidade composta de 471 funcionários com média de idade de 40 anos. Utilizou-se um questionário autoaplicável. Os resultados da atividade física mostram que o grupo $C$ obteve a maior média em todos os componentes e o grupo $B$ os menores valores com exceção da caminhada. Todos os participantes do estudo apresentaram bons escores nos quatro domínios da QV (58\% - 74\%). $\mathrm{Na}$ percepção de finitude, o grupo B obteve os melhores escores (percepção positiva) nos quatro dominios, enquanto os grupos $A$ e $C$ apresentaram uma percepção mais negativa, não havendo diferença significativa entre as variáveis. Evidencia-se a relação positiva da finitude na faixa etária dos 35 aos 50 anos. Foi identificado QV positiva e alto nível de atividade física.

Palavras-chave Finitude, Qualidade de vida, Idade 


\section{Introdução}

O processo de envelhecimento, a morte e a finitude são características intrínsecas do desenvolvimento humano, submetidos a um processo irreversível que inclui o nascer, o crescer e o morrer ${ }^{1}$. Além dos fatores relacionados com o desenvolvimento humano, os aspectos culturais, sociais, fisiológicos e psicológicos contribuem para uma visão diferenciada sobre a finitude humana ${ }^{2}$.

A percepção de finitude se inicia na infância ${ }^{3}$, mas é na adolescência que entendemos realmente o sentido da morte. Na idade adulta comprovamos tal fato como algo possível de acontecer, todavia é na velhice que sua possibilidade parece ser aceita, sendo encarada como a última etapa do ciclo do desenvolvimento humano no qual há um número maior de perdas, colaborando para que o idoso pense mais sobre sua finitude ${ }^{4,5}$. A perda de amigos e familiares, de sua ocupação, de parte de sua força física, redução do aparelho sensório e, em alguns casos, perda do funcionamento cerebral são comuns nesta idade ${ }^{6}$. A velhice pode aproximar a perspectiva da morte. Mesmo com o aumento da expectativa de vida da população humana, viver é um processo finito e esta finitude passa a ser mais decisiva com a chegada da velhice?

No entanto, é possível perceber a dificuldade em discorrer a respeito da finitude, em especial com os idosos, mesmo compreendendo que a cada dia que passa o ser humano está continuamente envelhecendo e morrendo ${ }^{8}$. Os mecanismos de defesa apresentados pelos indivíduos possibilitam que se ignore a morte e se dificulte a percepção da finitude do ser no mundo?.

Apresentar consciência de finitude, quanto mais cedo adquirida pode ajudar na compreensão da existência humana, percebendo-a como existência de possibilidade e importância de fazer escolhas para uma vida mais realizada ${ }^{10}$. À medida que o ser humano toma consciência de sua finitude, passa a compreender a vida em sua complexidade e tende a rever seus valores ${ }^{11}$.

Os trabalhos na área da saúde contribuem para o desenvolvimento de competências e habilidades relacionadas com a qualidade de vida ${ }^{12}$. No âmbito da saúde, seu sentido ampliado, se apoia na compreensão das necessidades humanas fundamentais e tem no conceito de promoção da saúde o seu foco mais relevante ${ }^{13}$.

Nas últimas décadas têm-se destacado a importância da aquisição e da manutenção de hábitos saudáveis visando à melhoria da qualidade de vida da população. A atividade física tem uma fundamental importância na qualidade de vida, associada ao bem estar e à saúde em todas as faixas etárias ${ }^{14-16}$. Independente de sexo, idade e profissão, é evidenciado que, para a melhora da qualidade de vida, a prática regular de atividade física é de grande valia. Pode-se observar que, em média, quanto mais ativa a pessoa, melhor sua qualidade de vida. Dentre as diferenças na qualidade de vida das pessoas que praticam atividade física comparadas com as que não praticam, não estão apenas os aspectos de saúde física, mas também aspectos psicológicos e cognitivos ${ }^{17}$.

Os estudos que abordam a temática finitude e qualidade de vida investigam mais amplamente temas relacionados à ética ${ }^{18}$, saúde pública ${ }^{12}$, enfermagem ${ }^{19,20}$ e medicina ${ }^{1}$. A relevância científica e social deste estudo consiste em apresentar um novo olhar acerca da finitude relacionada à idade. Para tanto, este analisou a influência da idade na percepção de finitude e qualidade de vida de funcionários públicos do Tribunal de Justiça de Santa Catarina.

\section{Métodos}

O estudo de corte transversal foi aprovado pelo Comitê de Ética em Pesquisa envolvendo Seres Humanos da Universidade do Estado de Santa Catarina que segue as Normas e Diretrizes Regulamentadoras da Pesquisa envolvendo Seres Humanos - Resolução CNS - 196/96²1.

A população do estudo foi composta de aproximadamente 1200 servidores públicos do Tribunal de Justiça de Santa Catarina, lotados em 2011. A fim de se abranger um maior número de trabalhadores, realizou-se um levantamento de instituições empregadoras ou prestadoras de serviços no nível da justiça, educação, saúde, desporto, religião, seguros e constatou- se que o Tribunal de Justiça era o local em que havia maior concentração de trabalhadores de ambos os sexos na idade a partir de 21 anos, sendo este escolhido para realização da coleta de dados.

O processo de amostragem foi o de conveniência, composto por 471 servidores de ambos os sexos com idade a partir de 21 anos, perfazendo uma média de $40 \pm 10,1$ anos, sendo $53 \%$ feminino.

Todos os participantes assinaram o termo de consentimento livre e esclarecido, concordando com a participação no estudo.

Para coleta de dados foi utilizado um questionário autoaplicável composto por 5 partes:

a) Identificação pessoal: nove perguntas para identificar sexo, idade, escolaridade, estado civil, ocupação funcional, doença e medicamentos; 
b) Situação socioeconômica: por meio do instrumento adotado pela Associação Nacional de Empresa de Pesquisa - Critério de Classificação Econômica Brasil ${ }^{22}$ que é considerado o principal instrumento de segmentação da população segundo seu poder de compra, o qual classifica a população em classes econômicas A, B, C, D e E, por meio da pontuação obtida e renda mensal;

c) Qualidade de Vida: WHOQOL (abreviado) que compõe os domínios físico, psicológico, social e ambiental (World Health Organization Quality of Life - WHOQOL). A versão em português do WHOQOL abreviado foi desenvolvido no Centro WHOQOL para o Brasil, no Departamento de Psiquiatria e Medicina Legal da Universidade Federal do Rio Grande do Sul em Porto Alegre no Estado do Rio Grande do Sul Brasil sob a coordenação geral do Dr. Marcelo Pio de Almeida Fleck ${ }^{23}$.

d) Questionário Internacional de Atividade Física (International Physical Activity Questionnaire) - IPAQ (versão curta). O IPAQ é um instrumento autoadministrável, composto por seis itens, que procura verificar o número de vezes em que o sujeito praticou pelo menos $10 \mathrm{minu}$ tos contínuos de caminhada, atividade física moderada e vigorosa, na última semana, em diversos envolvimentos, nomeadamente, laboral, doméstico, lazer, recreativo e desportivo ${ }^{24}$.

e) Perspectiva de envelhecimento pessoal composto pelo Inventário Sheppard de Atitudes em relação à velhice pessoal com a função de estimar as atitudes em relação à velhice. Adaptado semanticamente para o português e validado fatorialmente por $\mathrm{Neri}^{25}$. O instrumento é composto por 20 questões dividido em 4 subgrupos, os quais permitem avaliar a opinião do respondente quanto: a) possibilidade de ser feliz na velhice; b) se a velhice prenuncia dependência, morte e solidão; c) se é melhor morrer cedo do que sentir angústia e a solidão da velhice; d) se a velhice pode propiciar sentimentos de integridade. O mesmo é organizado em uma escala do tipo Likert de cinco pontos ("discordo muitíssimo" a "concordo muitíssimo") que permite dupla exploração dos resultados. Num primeiro momento, por meio de escores, é possível determinar se os participantes possuem uma percepção positiva ou negativa em relação à finitude (prevalência). Posteriormente, reorganizando a pontuação de 10 das 20 questões, pode-se calcular a média em cada um dos subgrupos sendo que quanto mais próxima de 1 (pontuação mínima) mais positiva a percepção do sujeito, e quanto mais próxima de 5 (pontuação máxima), mais negativa.
O instrumento foi autoaplicável e distribuído em todos os setores do Tribunal de Justiça de Santa Catarina, o qual foi respondido individualmente, com garantia de sigilo das respostas e identidade dos funcionários públicos.

Os dados foram analisados pelo pacote estatístico SPSS - 17.0, por meio da estatística descritiva (média, desvio padrão, amplitude e percentual) e inferencial pelo Teste do Qui Quadrado (comparação das características pessoais com os grupos etários) e ANOVA (domínios da qualidade de vida, finitude e atividade física com os grupos etários). O nível de significância estatística foi estabelecido para $\mathrm{p}<0.05$.

\section{Resultados}

A Tabela 1 está dividida por grupo etário, nomeadamente o grupo A (19-34 anos), grupo B (3550 anos) e grupo C (51-65 anos), no qual 55\% são do sexo feminino, destacando-se o grupo B com diferença significativa $(\mathrm{p}=0,003)$. No estado conjugal $63 \%$ moram juntos, prevalecendo o grupo C com $73 \%(\mathrm{p}=0,001)$, a maioria tem ensino superior completo ( $88 \%)$, são saudáveis $(81 \%)$ e não fazem uso de medicamentos $(86 \%)$, e pertencem ao estrato econômico B (63\%). O grupo A obteve maior percentual nestas variáveis $(\mathrm{p}=0,008 ; 0,001 ; 0,001 ; 0,050)$ respectivamente. Com relação ao nível de atividade física, $66 \%$ dos participantes são suficientemente ativos (ativo + muito ativo), destacando os sujeitos mais velhos (grupo $\mathrm{C}$ ), não havendo diferença significativa $(\mathrm{p}=0,448)$.

Observa-se na Tabela 2 que os participantes do estudo apresentaram bons escores nos quatro domínios da qualidade de vida $(58 \%-74 \%)$. O domínio social obteve a maior média (75\%) no grupo B, e o menor escore foi encontrado no domínio físico (58\%) também no grupo B, não havendo diferença significativa entre as variáveis $(\mathrm{p}=0,271-0,896)$.

A Tabela 3 apresenta os resultados da atividade física entre os diferentes grupos etários, no qual o grupo $\mathrm{C}$ obteve a maior média em todos os componentes da atividade física (caminhada - $54 \mathrm{~min} / \mathrm{d}$, atividade física moderada - $55 \mathrm{~min} / \mathrm{d}$; atividade física vigorosa $-34 \mathrm{~min} / \mathrm{d}$; atividade física moderada + vigorosa $-89 \mathrm{~min} / \mathrm{d}$ e atividade física total - $142 \mathrm{~min} / \mathrm{d}$ ) e o grupo B os menores valores com exceção da caminhada. Não havendo diferença significativa entre as variáveis.

A percepção da finitude entre os grupos etários é apresentada na Tabela 4, sendo que o gru- 
Tabela 1. Características dos participantes do estudo (\%).

\begin{tabular}{|c|c|c|c|c|c|}
\hline & Total & $\begin{array}{c}\text { Grupo } A \\
(19-34 \text { anos })\end{array}$ & $\begin{array}{c}\text { Grupo B } \\
(35-50 \text { anos })\end{array}$ & $\begin{array}{c}\text { Grupo C } \\
(51-65 \text { anos })\end{array}$ & Valor de $\mathrm{p}$ \\
\hline Sexo & & & & & 0,003 \\
\hline Masculino & 44,5 & 45,7 & 38,2 & 60,0 & \\
\hline Feminino & 55,3 & 54,3 & 61,8 & 38,7 & \\
\hline Estado conjugal & & & & & 0,001 \\
\hline Morando junto & 63,0 & 50,0 & 67,9 & 73,3 & \\
\hline Morando separado & 37,0 & 50,0 & 32,1 & 26,7 & \\
\hline Escolaridade & & & & & 0,001 \\
\hline Ensino fundamental & 1,4 & - & 0,9 & 5,3 & \\
\hline Ensino médio & 10,9 & 7,9 & 8,0 & 24,0 & \\
\hline Ensino completo & 87,8 & 92,1 & 91,0 & 70,7 & \\
\hline Doenças & & & & & 0,008 \\
\hline Sim & 18,2 & 10,0 & 21,6 & 24,0 & \\
\hline Não & 81,8 & 90,0 & 78,4 & 76,0 & \\
\hline Medicamentos & & & & & 0,001 \\
\hline $\operatorname{Sim}$ & 26,4 & 13,6 & 30,0 & 40,0 & \\
\hline Não & 73,6 & 86,4 & 70,0 & 60,0 & \\
\hline Estrato Econômico & & & & & 0,050 \\
\hline A & 22,0 & 17,1 & 25,8 & 20,0 & \\
\hline B & 63,6 & 68,6 & 62,9 & 56,0 & \\
\hline $\mathrm{C}$ & 10,0 & 11,4 & 7,5 & 14,7 & \\
\hline $\mathrm{D}$ & 4,4 & 2,9 & 3,8 & 9,3 & \\
\hline Nível de AF & & & & & 0,448 \\
\hline Insuficientemente ativo & 33,9 & 35,0 & 37,9 & 30,0 & \\
\hline Ativo & 38,3 & 42,9 & 33,1 & 39,0 & \\
\hline Muito ativo & 27,8 & 22,1 & 29,0 & 31,0 & \\
\hline
\end{tabular}

AF. Atividade Física

Tabela 2. Comparação da qualidade de vida entre os grupos etários (média \pm desvio padrão).

\begin{tabular}{lccccc}
\hline & Total & $\begin{array}{c}\text { Grupo A } \\
(\mathbf{1 9 - 3 4} \text { anos })\end{array}$ & $\begin{array}{c}\text { Grupo B } \\
(\mathbf{3 5 - 5 0} \text { anos })\end{array}$ & $\begin{array}{c}\text { Grupo C } \\
(\mathbf{5 1 - 6 5} \text { anos })\end{array}$ & Valor de p \\
\hline D. Físico (\%) & $58,1 \pm 9,9$ & $57,8 \pm 10,1$ & $57,7 \pm 10,0$ & $59,8 \pm 9,3$ & 0,271 \\
D. Psicológico (\%) & $64,8 \pm 10,8$ & $65,3 \pm 9,6$ & $67,0 \pm 11,2$ & $65,4 \pm 10,3$ & 0,336 \\
D. Social (\%) & $73,9 \pm 15,1$ & $74,7 \pm 14,9$ & $74,8 \pm 18,4$ & $74,4 \pm 15,6$ & 0,896 \\
D. Ambiental (\%) & $65,1 \pm 13,8$ & $66,3 \pm 13,0$ & $66,9 \pm 14,5$ & $66,0 \pm 13,5$ & 0,569
\end{tabular}

D. Domínio

Tabela 3. Comparação da atividade física entre os grupos etários (média \pm desvio padrão).

\begin{tabular}{lccccc}
\hline & Total & $\begin{array}{c}\text { Grupo A } \\
(\mathbf{1 9 - 3 4} \text { anos })\end{array}$ & $\begin{array}{c}\text { Grupo B } \\
(\mathbf{3 5 - 5 0} \text { anos })\end{array}$ & $\begin{array}{c}\text { Grupo C } \\
(\mathbf{5 1 - 6 5} \text { anos })\end{array}$ & $\begin{array}{c}\text { Valor } \\
\text { de } \mathbf{~ p}\end{array}$ \\
\hline Caminhada (min/d) & $49,6 \pm 46,8$ & $44,2 \pm 44,1$ & $51,6 \pm 47,1$ & $54,4 \pm 50,8$ & 0,224 \\
A.F. Moderada (min/d) & $50,8 \pm 56,2$ & $50,1 \pm 55,0$ & $49,7 \pm 54,9$ & $55,2 \pm 62,2$ & 0,765 \\
A.F Vigorosa (min/d) & $31,7 \pm 48,7$ & $33,0 \pm 48,8$ & $30,1 \pm 46,5$ & $33,8 \pm 54,9$ & 0,794 \\
A.F Moderada + Vigorosa (min/d) & $82,6 \pm 83,3$ & $82,3 \pm 80,1$ & $80,4 \pm 81,0$ & $89,5 \pm 95,5$ & 0,726 \\
A.F Total & $1131,8 \pm 107,4$ & $127,7 \pm 103,7$ & $131,2 \pm 106,8$ & $141,6 \pm 116,6$ & 0,674 \\
\hline
\end{tabular}


po B obteve os melhores escores (percepção positiva) nos quatro domínios, (mais próximos de um), enquanto os grupos A e C apresentaram escores mais próximos de cinco retratando uma percepção mais negativa quanto à finitude, não havendo diferença significativa entre as variáveis.

De um modo geral os resultados da Tabela 5, revelam associações positivas com o domínio físico e dependência $\left(r=0,095^{*}\right)$ e domínios psicológico, social e ambiental com integridade e velhice $\left(r=0,140^{\star *}, 0,120^{\star *}, 0,116^{*}\right)$ respectivamente, demonstrando que à medida que os escores da qualidade de vida nestes domínios aumentam há uma percepção mais positiva da finitude quanto à dependência e integridade na velhice. Associações negativas também foram verificadas entre alguns domínios da qualidade de vida e finitude. A morte foi associada negativamente com o domínio psicológico $\left(\mathrm{r}=-0,121^{\star \star}\right)$ e domínio ambiental $\left(r=-0,136^{* *}\right)$ e a dependência com os domínios psicológico $\left(r=-0,206^{\star *}\right)$, social $(r=-$ $\left.0,122^{\star *}\right)$ e ambiental $\left(r=-0,123^{\star *}\right)$.

\section{Discussão}

A sociedade marcada por um ritmo alucinante parece ter deixado de lado o fato de todos sermos seres finitos. Assim, o homem tende a não pensar sobre sua finitude e a das pessoas que o rodeiam. Nota-se um despreparo no que diz respeito ao enfrentamento dessa situação ${ }^{26}$.
No nosso estudo não foram encontradas diferenças significativas entre as variáreis relacionadas à finitude nos três grupos etários, mas parece haver uma tendência dos participantes a acreditarem numa perspectiva de integridade na velhice mais positiva. Porém, na faixa etária dos 35-50 anos, encontrou-se uma percepção mais positiva em relação às demais idades estudadas.

A variável dependência na velhice foi mais negativa em indivíduos mais jovens quando comparados a indivíduos mais velhos. Em controvérsia, num estudo feito por Zanon ${ }^{27}$, observou uma similaridade com relação à percepção positiva na velhice tanto entre os jovens como nos idosos (48\%). Outros estudos verificaram que em idades mais avançadas, tem-se uma percepção mais positiva em relação à velhice, destacando a valorização com a família, a independência financeira, bem como a capacidade em realizar as suas atividades ${ }^{28,29}$.

Araújo e Carvalho ${ }^{30}$ investigando indivíduos com média de idade de 65 anos apontaram para uma significativa negação da velhice, sobretudo naqueles com maior poder aquisitivo, o que difere de nosso estudo, pois assim como os demais grupos etários, a maioria dos participantes do grupo C (51-65 anos), pertencem ao estrato econômico B (56\%), apresentando resultados satisfatórios com relação à finitude em todas as suas variáveis.

É relevante ressaltar que a população do presente estudo possui um nível de escolaridade alto,

Tabela 4. Comparação da percepção dos domínios da finitude entre os grupos etários (média \pm desvio padrão).

\begin{tabular}{lccccc}
\hline & Total & $\begin{array}{c}\text { Grupo A } \\
(\mathbf{1 9 - 3 4} \text { anos })\end{array}$ & $\begin{array}{c}\text { Grupo B } \\
(\mathbf{3 5 - 5 0} \text { anos })\end{array}$ & $\begin{array}{c}\text { Grupo C } \\
(\mathbf{5 1 - 6 5} \text { anos })\end{array}$ & $\begin{array}{c}\text { Valor } \\
\text { de } \mathbf{p}\end{array}$ \\
\hline Felicidade e velhice (x) & $2,2 \pm 0,6$ & $2,3 \pm 0,5$ & $2,2 \pm 0,7$ & $2,3 \pm 0,6$ & 0,461 \\
Dependência e velhice (x) & $3,0 \pm 1,1$ & $3,9 \pm 1,0$ & $2,9 \pm 1,2$ & $3,0 \pm 1,0$ & 0,618 \\
Morte e velhice (x) & $3,9 \pm 1,2$ & $3,9 \pm 1,1$ & $3,8 \pm 1,3$ & $3,9 \pm 1,1$ & 0,740 \\
Integridade e velhice (x) & $1,5 \pm 0,9$ & $1,5 \pm 0,9$ & $1,3 \pm 0,8$ & $1,8 \pm 1,3$ & 0,005 \\
\hline
\end{tabular}

Tabela 5. Associação entre os domínios da finitude e qualidade de vida dos participantes do estudo.

\begin{tabular}{lcccc}
\hline & Felicidade e velhice & Dependência e velhice & Morte e velhice & Integridade e velhice \\
\hline D. Físico & 0,031 & $0,095^{*}$ & $-0,044$ & 0,088 \\
D.Psicológico & 0,070 & $-0,206^{* *}$ & $-0,121^{* *}$ & $0,140^{* *}$ \\
D. Social & 0,048 & $-0,122^{* *}$ & $-0,047$ & $0,120^{* *}$ \\
D. Ambiental & 0,059 & $-0,123^{* *}$ & $-0,136^{* *}$ & $0,116^{*}$ \\
\hline
\end{tabular}

D. domínio; Correlação de Spearman; ${ }^{*} \mathrm{p}=0,05 ;{ }^{* *} \mathrm{p}=0,01$ 
o que pode trazer maior entendimento quanto à necessidade de manter um estilo de vida saudável, por meio da prática de atividade física. Diferenças na condição financeira, na oportunidade de acesso aos recursos e às orientações para a saúde ${ }^{31-33}$, refletem em um bom nível de qualidade de vida e consequentemente em uma percepção mais positiva com relação à velhice ${ }^{34}$.

Neste estudo, a maioria dos participantes mostrou-se suficientemente ativa (ativo + muito ativo), destacando-se o grupo C (composto dos indivíduos mais velhos), assemelhando-se aos achados de Burton e Turrell ${ }^{35}$ e Al-hazzaa ${ }^{36}$. Observam-se na literatura alguns dados contraditórios, uma vez que no estudo de Brito et al. ${ }^{37}$ os indivíduos mais velhos obtiveram baixo nível de atividade física. Enquanto Hallal et al. ${ }^{38}$ aponta os adultos jovens como sendo os mais ativos, e finalmente Carlson et al. ${ }^{39}$ e Matsudo et al..$^{33}$ mostram não haver diferenças relevantes nos níveis de atividade física entre diferentes faixas etárias.

Estudos atuais têm demonstrado impactos positivos da prática regular de atividade física contribuindo para a saúde e o bem-estar da população em geral ${ }^{40-43}$. Como exemplos destes impactos destacam-se os benefícios físicos, psicológicos e sociais ${ }^{16,44}$.

A atividade física/exercício físico parece influenciar favoravelmente a qualidade de vida relacionada com a saúde, independentemente da idade e do gênero ${ }^{45-49}$. A atividade física representa ainda um impacto positivo na qualidade de vida, independente da idade, do sexo e do estado de saúde ${ }^{50,51}$. Nesse sentido, Gonçalves et al. ${ }^{52}$ verificaram que a prática de atividade física apresenta relevância para a saúde física e mental, atuando nos principais domínios da vida. Há pouca informação sobre a atividade física e seus efeitos nos diversos domínios da qualidade de vida relacionada com a saúde. Trata-se de um aspecto relevante, uma vez que diferentes quantidades e/ ou intensidades de exercício físico podem influenciar diferentemente os diversos domínios da qualidade de vida. Por outro lado, a recomendação para a prática de atividade física no âmbito de saúde pública considera a atividade física acumulada semanalmente $(150 \mathrm{~min} / \mathrm{semana}$ de intensidade pelo menos moderada) e não somente o exercício formal ${ }^{53}$. Em nosso estudo os participantes realizaram mais atividade física do que o recomendado para esta população (adultos), ou seja, $132 \mathrm{~min} /$ dia vs $150 \mathrm{~min} /$ semana.
Os participantes deste estudo apresentaram bons escores nos quatro domínios da qualidade de vida, corroborando com Pucci et al. ${ }^{54}$. A qualidade de vida esta relacionada às necessidades biológicas, sendo uma somatória de fatores decorrentes da interação entre saúde, alimentação, ambiente, moradia, recursos econômicos, relacionamentos, lazer e fatores de satisfações psicológicas, autoestima, identidade e espiritualidade ${ }^{55-59}$.

No presente estudo a qualidade de vida esteve relacionada com a percepção de finitude da amostra, demonstrando que à medida que os escores da qualidade de vida no domínio psicológico, social e ambiental aumentam, há uma percepção mais positiva da finitude quanto à dependência e integridade na velhice. Semelhantemente um estudo com idosos com média de idade de 63 anos identificou que a amostra de um modo geral apresentou escores positivos na qualidade de vida, sobretudo no domínio ambiental, e quando avaliada a finitude esta resultou em uma percepção positiva destacando-se a percepção de integridade na velhice ${ }^{60}$, sendo possível elucidar que assim como no presente estudo a qualidade de vida neste caso influenciou de maneira positiva a percepção de finitude dos idosos.

Entretanto também foi observado nos participantes do nosso estudo que à medida que os escores da qualidade de vida nos domínios psicológico e ambiental diminuem há uma percepção mais negativa quanto à morte e a dependência, corroborando com a literatura, uma vez que a dependência caracterizada muitas vezes pela incapacidade funcional é resultante da interação entre componentes psicológicos e ambientais da vida do indivíduo, sendo que quando estes domínios estão em um nível baixo, o indivíduo se encontra mais suscetível a apresentar dependência na velhice e consequentemente uma visão mais negativa quanto à finitude ${ }^{61}$.

Neste contexto a percepção da qualidade de vida e da finitude também pode ser influenciada por fatores socioculturais como o trabalho. Quando este deixa de ser vivenciado - pelo fato da aposentadoria ou pelo desemprego - compromete a qualidade do envelhecimento, principalmente se faltarem habilidades e condições socioeconômicas, para priorizar outras atividades e valores ${ }^{62}$. Podemos elucidar que os participantes do estudo por serem na sua maioria casados, com ensino superior completo, com ausência de doenças, do estrato econômico B, além de serem suficientemente ativos legitimam a qualidade de vida positiva, talvez por estarem empregados e terem a garantia da aposentadoria. 


\section{Considerações finais}

A principal contribuição deste estudo refere-se à investigação entre a idade e a percepção de finitude e qualidade de vida de funcionários públicos. Os resultados põem em evidência a relação positiva da finitude na faixa etária dos 35 aos 50 anos. Nos três grupos etários observou-se uma percepção negativa em relação à morte e velhice. Enquanto o grupo mais jovem (19 a 34 anos) apresentou também uma percepção negativa quanto à dependência e morte. Foi identificado entre os participantes do estudo uma qualidade de vida positiva nos quatro domínios, influenciando na percepção de finitude, além do alto nível de atividade física, superior ao recomendado para a população adulta.

Por fim, cabe ressaltar a necessidade de novas pesquisas que avaliem os aspectos trabalhados neste estudo, estendendo-os a outras populações e contextos socioculturais, envolvendo maior número de participantes a fim de verificar a generalização destes dados.

\section{Colaboradores}

CCR Araujo, ACA Guimaraes, C Meyer, L Boing, MO Ramos, MC Souza e SR Parcias trabalharam na concepção, delineamento, análise e interpretação dos dados; redação do artigo e aprovação da versão submetida. 
1. Schramm FR. Morte e finitude em nossa sociedade: implicações no ensino dos cuidados paliativos. Rev Bras Cancerol 2002; 48(1):17-20.

2. Combinato DS, Queiroz MS. Morte: uma visão psicossocial. Estud Psicol 2006; 11(2):209-216.

3. Papalia DE, Olds SW. Desenvolvimento Humano. Porto Alegre: Artes Médicas; 2000.

4. Hohendorff JV, Melo WV. Compreensão da morte e desenvolvimento Humano: contribuições à Psicologia Hospitalar. Estud Pesqui Psicol 2009; 9(2):480492.

5. Kovács MJ. Educação para morte. Sugestões de linhas de ações para o psicólogo. São Paulo: Casa do Psicólogo; 2005.

6. Silva CA, Carvalho LS, Santos ACPO, Menezes MR. Vivendo após a morte de amigos: história oral de idosos. Texto Contexto Enferm 2007; 16(1):97-104.

7. Soares JA, Silva RF, Rosa LJ, Ribeiro RN. O idoso institucionalizado e a reflexão sobre a própria morte. Rev Kairós 2009; 12(1):135-147.

8. Benincá CRS. Idoso e morte: qualificação da experiência de finitude. In: Both A, Barbosa MHS; Benincá CRS, organizadores. Envelhecimento humano: múltiplos olhares. Passo Fundo (RS): UPF; 2003. p. 82-95.

9. Lunardi ZM. Convivendo com a morte e o morrer no cotidiano da unidade de terapia intensiva [dissertação]. Erechim (RS): Universidade Regional Integrada do Alto Uruguai e das Missões; 2004.

10. Martins AA. Consciência da finitude, sofrimento e espiritualidade. Mundo Saúde 2007; 31(2):174-178.

11. Frumi C, Celich KLS. O olhar do idoso frente ao envelhecimento e à morte. Rev Bras Ciên Envelh Hum 2006; 3(2):92-100.

12. Rego S, Palácios M. A finitude humana e a saúde pública. Cad Saude Publica 2006; 22(8):1755-1760.

13. Minayo MCS, Hartz ZM de A, Buss PM. Qualidade de vida e saúde: um debate necessário. Cien Saude Colet 2000; 5(1):7-18.

14. Nahas MV. Atividade física, saúde e qualidade de vida: Conceitos e sugestões para um estilo de vida ativo. 2a Edição. Londrina: Midiograf; 2006.

15. Tassitano R, Bezerra J, Tenório M, Colares M, Barros V, Hallal PC. Atividade Física em adolescentes Brasileiros: Uma revisão Sistemática. Rev Bras Cineantropom Desempenho Hum 2007; 9(1):55-60.

16. Boiché JCS, Sarrazin PG. Self-determination of contextual motivation, inter-context dynamics and adolescents patterns of sport participation over time. Psychol Sport Exerc 2007; 8(5):685-703.

17. Silva RS, Silva I, Silva R, Souza L, Tomasi E. Atividade física e qualidade de vida. Cien Saude Colet 2010; 15(1):115-120.

18. Loparic Z. A ética da finitude. In: Benedito N, organizador. A crise do pensamento. Belém: Editora Universitária; 1994.

19. Takahashi CB, Contrin LM, Beccaria LM, Goudinho MV, Pereira RAM. Morte: percepção e sentimentos de acadêmicos de enfermagem. Arq Ciências Saúde 2008; 15(3):132-138.

20. Bernieri J, Hirdes A. O preparo dos acadêmicos de enfermagem brasileiros para vivenciarem o processo morte-morrer. Texto Contexto Enferm 2007; 16(1):89-96.
21. Brasil. Ministério da Saúde (MS). Conselho Nacional de Saúde. Resolução nº 196 de 10 de outubro de 1996. Diretrizes e Normas Regulamentadoras de Pesquisas Envolvendo Seres Humanos. Diário Oficial da União 1996; 16 out.

22. Associação Brasileira de Estudos Populacionais (ABEP). Critério Padrão de Classificação Econômica Brasil. Novo Critério Padrão de Classificação Econômica Brasil. Belo Horizonte: ABEP; 2008.

23. Fleck MPA, Louzada S, Xavier M, Chachamovic E, Vieira G, Santos L, Pinzon V. Aplicação da versão em português do instrumento de avaliação de qualidade de vida da OMS. (Whoqol - 100). Rev Saude Publica 1999; 33(2):198-205.

24. Pardini R, Matsudo SMM, Matsudo VKR, Araujo T, Andrade E, Braggion G. Validation of international physical activity questionnaire (IPAQ): pilot study in brazilian young adults. Med Sci Sports Exerc 1997:S5-S9.

25. Neri AL. O inventário Sheppard para medida de atitudes em relação à velhice e sua adaptação para o português. Est Psicol 1986; 3(1 e 2):23-42.

26. Fidelis WMZ. A morte e o morrer nas representações sociais dos alunos de curso de ensino médio de enfermagem [tese]. São Paulo (SP): Universidade de São Paulo; 2001.

27. Zanon CBFM. A educação e a intergeracionalidade na perspectiva da inserção social do idoso: desafios e possibilidades [dissertação]. Brasília (DF): Universidade Católica de Brasília; 2006.

28. Jardim VCFS, Medeiros BF, Brito AM. Um olhar sobre o processo do envelhecimento: a percepção de idosos sobre a velhice. Rev Bras Geriatr Gerontol 2006; 9(2):25-34.

29. Levy BR, Slade MD, Kunkel SR, Kasl SV. Longevity Increased by Positive Self-Perceptions of Aging. $J$ Pers Soc Psychol 2002; 83(2):261-270.

30. Araújo LF, Carvalho VAM. Velhices: Estudo Comparativo das Representações Sociais entre Idosos de Grupos de Convivências. Text sobre Envelhec 2004; 1(6):57-75.

31. Caspersen CJ, Zack MM. The prevalence of physical inactivity in the United States. In: Physical Activity and Cardiovascular health. A national Consensus Human Kinetics; 1997.

32. Martinez-Gonzalez MA, Varo JJ, Santos JL, De Irala J, Gibney M, Kearney J, Martinez JA. Prevalence of physical activity during leisure time in Europe Union. Med Sci Sports Exerc 2001; 33(7):1142-1146.

33. Matsudo SM, Matsudo VR, Araújo T, Andrade D, Oliveira L, Braggion G. Nível de atividade física da população do estado de São Paulo: análise de acordo com o gênero, idade, nível socioeconômico, distribuição geográfica e de conhecimento. Rev Bras Ciên Mov 2002; 10(4):41-50.

34. Mota J, Ribeiro JL, Carvalho J, Matos MG. Atividade física e qualidade de vida associada à saúde em idosos participantes e não participantes em programas regulares de atividade física. Rev Bras Educ Fís Esporte 2006; 20(3):219-225.

35. Burton NW, Turrell G. Occupation, Hours Worked, and Leisure-Time Physical Activity. Prev Med 2000; 31(6):673-681. 
36. Al-Hazzaa HM. Health-enhancing physical activity among Saudi adults using the International Physical Activity Questionnaire (IPAQ).J Public Health Nut 2007; 10(1):59-64.

37. Brito WF, Santos CL, Marcolongo AA, Campos MD, Bocalini DS, Antonio EL, Junior JAS, Tucci PJF, Serra AJ. Physical activity levels in public school teachers. Rev Saude Publica 2012; 46(1):104-109.

38. Hallal PC, Matsudo SM, Matsudo VKR, Araújo TL, Andrade DR, Bertoldi AD. Physical activity in adults from two Brazilian areas: similarities and differences. Cad Saude Publica 2005; 21(2):572-580.

39. Carlson SA, Fulton JE, Schoenborn CA, Loustalot F. Fleetwood. Trend and Prevalence Estimates Based on the 2008 Physical Activity Guidelines for Americans. Am J Prev Med 2010; 39(4):305-313.

40. Shibata A, Oka K, Nakamura Y, Muraoka I. Prevalence and demographic correlates of meeting the physical activity recommendation among Japanese adults. Phys Act Health 2009; 6(1):24-32.

41. Siqueira FV, Facchini LA, Piccini RX, Tomasi E, Thumé E, Silveira DS, Hallal PC. Atividade física em adultos e idosos residentes em áreas de abrangência de unidade básicas de saúde de municípios das regiões Sul e Nordeste do Brasil. Cad Saude Publica 2008; 24(1):39-54.

42. Matsudo SMM. Envelhecimento, atividade física e saúde. Bolet Inst Saúde 2009; 47:76-79.

43. Nelson ME, Rejeski J, Blair SN, Duncan PW, Judge JO, King AC, Macera CA, Sceppa CC. Physical Activity and Public Health in Older Adults: Recommendation From the American College of Sports Medicine and the American Heart Association. Jam Heart Assoc 2007; 116(9):1094-1105.

44. Haskell WL, Lee I-MIN, Pate RR, Powell KE, Blair, SN, Franklin BA, Macera CA, Heath GW, Thompson PD, Bauman A. Physical activity and public health: Updated Recommendations for Adults from The American College of Sports Medicine and The American Heart Association. Jam Heart Assoc 2007; 116(9):1081-1093.

45. Sguizzatto GT, Garcez-Leme LE, Casimiro L. Evaluation of the quality of life among elderly female athletes. São Paulo Med J 2006; 124(5):304-305.

46. Spirduso WW, Cronin DL. Exercise dose-response effects on quality of life and independent living in older adults. Med Sci Sports Exerc 2001; 33(Supl. 6):598-608.

47. King AC, Pruitt LA, Phillips W, Oka R, Rodenburg A, Haskell WL. Comparative effects of two physical activity programs on measured and perceived physical functioning and other health-related quality of life outcomes in older adults. Journal Geront 2000; 55(2):74-83.

48. Dorn J, Vena J, Jonh EM, Horn-Ross JL, Koo J. Lifetime physical activity and breast cancer risk in pre and postmenopausal women. Med Sci Sports Exerc 2003; 35(2):278-285.

49. Wolin KY, Colditz GA, Glynn R, Lee I-Min, Kawachi I. Long-term physical activity and health-related quality of life in middle aged women. Med Sci Sports Exerc 2005; 37(5):45-55.
50. Potter R, Ellard D, Rees K, Thorogood M. A systematic review of the effects of physical activity on physical functioning, quality of life and depression in older people with dementia. Intern Journal Geriat Psyc 2011; 26(10):1000-1011.

51. Guedes DP, Hatmann AC, Martini FA, Borges MB, Bernardelli Júnior R. Quality of life and physical activity in a sample of Brazilian older adults. J Aging and Health 2011; 24(2):212-226.

52. Gonçalves AKS, Canário ACG, Cabral PUL, Silva RAH, Spyrdes MHC, Giraldo PC, Eleutério Júnior J. Impacto da atividade física na qualidade de vida de mulheres de meia idade: estudo de base populacional. Rev Bras Ginecol Obstet 2011; 33(12): 408-413.

53. World Health Organization (WHO). Steps to Health: A European Frame Work to Promote Physical Activity for Health. Copenhagen: WHO; 2007.

54. Pucci GCMF, Rech CR, Fermino RC, Reis RS. Associação entre atividade física e qualidade de vida em adultos. Rev Saude Publica 2012; 46(1):166-179.

55. Campos MO, Neto JFR. Qualidade de vida um instrumento para promoção da saúde. Rev Ba Saúde Públ 2008; 32(2):232-240.

56. Lourenção LG, Moscardini AC, Soler ZASG. Saúde e qualidade de vida de médicos residentes. Rev Ass Med Bras 2010; 56(1):81-91.

57. Gerealds CF, Grillo DE, Merida M, Souza JX, Campanell JR. Nível de qualidade de vida dos colaboradores da diretoria de esportes e lazer do SESI- SP. Rev Macken Ed Física 2006; 5(3):13-22.

58. Aguiar MIF, Farias DR, Pinheiro ML, Chaves ES, Rolim ILTP, Almeida PC. Qualidade de vida de pacientes submetidos ao transplante cardíaco: aplicação da escala whoqol-bref. Arq Bras Cardiol 2011; 96(1):60-67.

59. Noce F, Simim MAM, Mello MT. A percepção de qualidade de vida de pessoas portadoras de deficiência física pode ser influenciada pela prática de atividade física? Rev Bras Med Esporte 2009; 15(3):174-178.

60. Guimarães ACA, Scotti AV, Soares A, Fernandes S, Machado Z. Percepção da qualidade de vida e da finitude de adultos de meia idade e idoso praticantes e não praticantes de atividade física. Rev Bras Geriatr Gerontol 2012; 15(4):661-670.

61. Miguel MEGB, Pinto MEB, Marconi SS. A dependência na velhice sob a ótica de cuidadores formais de idosos institucionalizados. Rev Eletrônica de Enferm 2007; 9(3):784-795.

62. Souza RF, Matias HA, Bretas, ACP. Reflexões sobre envelhecimento e trabalho. Cien Saude Colet 2010; 15(6):2835-2843.

Artigo apresentado em 22/03/2013

Aprovado em 22/05/2013

Versão final apresentada em 29/05/2013 